\title{
Design of a Damping Controller Using the SCA Optimization Technique for the Improvement of Small Signal Stability of a Single Machine Connected to an Infinite Bus System
}

\author{
Abdul Waheed Khawaja 1,2,*, Nor Azwan Mohamed Kamari 1,*(D) \\ and Muhammad Ammirrul Atiqi Mohd Zainuri ${ }^{1}$ D \\ 1 Department of Electrical, Electronic \& System Engineering, Faculty of Engineering and Built Environment, \\ Universiti Kebangsaan Malaysia, Bangi 43600, Selangor, Malaysia; ammirrulatiqi@ukm.edu.my \\ 2 Department of Electrical Engineering, Faculty of Engineering \& Technology, Bahauddin Zakariya University, \\ Multan 60800, Punjab, Pakistan \\ * Correspondence: khawaja@bzu.edu.pk (A.W.K.); azwank@ukm.edu.my (N.A.M.K.)
}

check for

updates

Citation: Khawaja, A.W.; Kamari, N.A.M.; Zainuri, M.A.A.M. Design of a Damping Controller Using the SCA Optimization Technique for the Improvement of Small Signal Stability of a Single Machine Connected to an Infinite Bus System. Energies 2021, 14 2996. https://doi.org/10.3390/ en14112996

Academic Editor: Andrea Mariscotti

Received: 3 April 2021

Accepted: 18 May 2021

Published: 21 May 2021

Publisher's Note: MDPI stays neutral with regard to jurisdictional claims in published maps and institutional affiliations.

Copyright: (c) 2021 by the authors. Licensee MDPI, Basel, Switzerland. This article is an open access article distributed under the terms and conditions of the Creative Commons Attribution (CC BY) license (https:// creativecommons.org/licenses/by/ $4.0 /)$.

\begin{abstract}
This paper discusses the use of a stochastic metaheuristic population-based optimization algorithm known as the sine cosine algorithm (SCA) to design the parameters of a power system stabilizer (PSS) for damping electromechanical oscillations in a single machine connected to a large power system. The design of the PSS parameters was formulated as an optimization problem to minimize the objective function value. The SCA was used to obtain the best values of the PSS parameters under the objective function. Simulation was carried out by a linearized power system model. The lead lag controller was used as the PSS structure and the results from that were compared with those obtained by moth flame optimization and evolutionary programming. The results showed that the SCA is more effective than are the other techniques in exploration and exploitation to tune the PSS parameters and enhance the power system stability by damping oscillations in a range of loading conditions.
\end{abstract}

Keywords: power system stability; sine cosine algorithm; eigenvalues; objective function; power system stabilizer parameters; single machine infinite bus

\section{Introduction}

The main source of electrical power is a synchronous generator. The electric power system is complex, and a stable power system operates in equilibrium. In large-interconnected power systems, the variation in load and generation initiates rotors of synchronous machines to swing, causing changes in machine rotor angles, terminal voltages, and frequencies that affect the power system equilibrium. Furthermore, the efficiency of alternators, long transmission lines, distribution lines, and all appliances connected to the power system are affected by these changing characteristics. After a perturbation, synchronous machines attempt to regain equilibrium but at different rotor angles. Therefore, oscillations that are not damped completely may lead to an increase in low frequency oscillations in power networks, causing problems in system stability and reducing the power transfer capacity of transmission lines [1-3]. The power system's low frequency oscillations are classified into two types, one with local area modes of oscillation that have a frequency range from 0.8 to $3 \mathrm{~Hz}$ and the other with inter-area modes of oscillations that range from 0.2 to $0.7 \mathrm{~Hz}[4]$.

The power system stability can be mainly divided into three types, namely, voltage, angle, and frequency. Voltage stability is the power system's ability to maintain the required acceptable voltages at all nodes after perturbation, such as a change in active and reactive load demands under normal operating conditions. The change in node voltages due to active and reactive power demands are associated with the rotor angles, and is 
a local phenomenon, but it has widespread effects on a significant portion of the power system and can lead to voltage collapse. Angle stability is the ability of a power system's synchronous machines to remain in a synchronized state or regain synchronism after being subjected to a physical disturbance. Any variations in load cause changes in rotor speed of the synchronous generator that further affect the generated output and rotor angle at different synchronous rotor speeds. Under equilibrium conditions, the rotor speed has synchronous speed and machines have a balance between electromagnetic and mechanical torques. Changes in rotor speed, rotor angle, and generated output cause synchronous machines to have oscillations in power networks due to an imbalance in the mechanical and electromagnetic torques [1]. Frequency stability is the ability of a power system to maintain the required, acceptable frequency against large variations in load or generation. These variations include large loads suddenly being connected to or disconnected from a power system and large generating units being disconnected by a protection system, creating a power imbalance between what is delivered by the turbines and what is consumed by the load [2].

In effectively dampening the electromechanical oscillations, the formulation of the objective function plays a key role by using a combination of indicators of the power system. In angle stability analysis, creating an objective function that efficiently locates the eigenvalues in a more stable region in the complex s-plane is important. The indicators that assess the angle stability are the damping ratio and a factor of the eigenvalues, determined from imaginary and real eigenvalues of complex power systems. The angle stability can be assessed using a damping factor that improves results by shifting eigenvalues towards the left of the imaginary axis of the complex s-plane using formulations of the maximized real parts of each eigenvalue as an objective function of minimization. The damping ratio shifts the eigenvalues towards the real axis of the complex s-plane using formulations of the minimum damping ratio of each eigenvalue as an objective function of maximization [5].

Proper implementation of damping devices is required to dampen oscillations and thus maintain or improve the power system's stability. Different schemes and modelling approaches have been used for damping, such as power system stabilizers (PSSs), flexible alternating current transmission systems (FACTS), and coordination control. The PSS controller, with the excitation system of synchronous machine controls, outputs power by providing the additional synchronizing torque in phase with speed deviations to dampen the required oscillations and improve the power angle stability [1,4].

The stability of a system is a primary concern because synchronous machines should remain in a synchronous state while working with other machines in a group or with generators of other regions to form large-interconnected power systems. Perturbation in the system produces low frequency oscillations and affects electrical generation that could lead to unstable machines that trip other units and collapse the system. Application of a PSS is a first measure to enhance the small signal stability. A PSS is the preferred method to improve the power system damping effect [6,7]; its installation is economical and effective for stabilizing low frequency oscillations within a system $[6,8,9]$.

Different types of FACTS devices—series, shunt, series shunt, and series-series-have been used to dampen oscillations in power systems through proper design of controllers by injecting or absorbing reactive power. Two FACTS shunt devices, static VAR compensators (SVC) and static synchronous compensators (STATCOM), have been used for damping to enhance small signal stability [10]. A damping performance analysis [11], dynamic control strategy to improve the power flow capabilities [12], and enhancement of the low voltage ride through capability in power systems [13] have also been described in the literature. In coordination control, PSS and FACTS devices with efficient coordination among controllers have been combined to dampen both types of oscillations in power systems, namely, local and inter-mode. The coordination control design for the thyristor-controlled series capacitor (TCSC), SVC, and PSS [14,15] and of the PSS and series capacitive reactance compensator (SCRC) were used to enhance damping [16]. Furthermore, STATCOM has 
been coordinated with PSS-STATCOM and a unified power flow controller (UPFC), and UPFC has been coordinated with a PSS [17-20].

Optimization techniques have been widely used to calculate the suitable parameters of PSS and FACTS-based controllers. The controller parameters are tuned by optimization techniques, such as conventional, deterministic, heuristic, and hybrid. The heuristic technique solves the optimization problems using stochastic methods, and its improved techniques are known as metaheuristic algorithms. Various metaheuristic optimization techniques have been used to tune parameters of controllers of PSS and FACTS devices [4]. The genetic algorithm (GA) was used to design a robust PSS and a coordinated PSS with UPFC [21-23]. The particle swarm optimization (PSO) algorithm was used to design a damping controller $[5,24,25]$, as were cuckoo and BAT optimization algorithms [26,27]. The firefly optimization algorithm was used to design an SVC damping controller [28,29]. Various heuristic algorithms for optimization have used stochastic methods that have deficiencies in solution convergence. GA and PSO have deficiencies in convergence, that is, a local minimum stagnation problem. Several proposed methods provide good exploration in a search space but are limited in exploitation, or vice versa. The main advantage of the sine cosine algorithm (SCA) is in allowing both exploration and exploitation in a search space, that is, for the first and second halves of the iterations, respectively.

This research aimed to develop a model of a machine connected to large system and formulate the objective function based on a maximum damping factor to improve the angle stability of the power system. The PSS was chosen as the proposed device to improve the damping capability of the power system. For an efficient control capability of the PSS, the lead lag controller parameters were optimized. For comparison purposes, the performance of the PSS lead lag controller was tuned with the SCA and the resulting parameters were compared with those optimized with moth flame optimization (MFO) and evolutionary programming (EP) to accurately and effectively predict and assess the angle stability before a power system collapse.

This study presents the application of metaheuristic optimization algorithms and the objective function and then proposes a design for a robust controller of the excitation of a machine-connected infinite bus system. The objective function was formulated as a maximum value of the real part as an indicator, and the SCA was developed to tune the lead lag controller parameters. $T_{1}, T_{2}$, and $T_{W}$ are time constants for phase compensation and washout of the PSS under different loading conditions to improve damping efficiency in angle stability for a single machine infinite bus (SMIB) system. For comparison of optimization techniques, the PSS controller optimized with the SCA method was compared with the PSS parameters tuned with the MFO and EP algorithms.

In this paper, the small signal stability of a power system was improved by mathematical modelling of a SMIB and formulation of an objective function based on a maximum damping factor. The key highlights of the paper are as follows:

- Novel machine parameters utilizing flux in the amortisseur windings are considered in the proposed model of the SMIB system.

- $\quad$ The proposed SMIB model linearizes the state space model matrix and a $9 \times 9$ dimension is obtained that is applied in the eigenvalue analysis.

- The SCA optimization technique shows improvement in exploration and exploitation processes and finds maxima or minima in the eigenvalue analysis.

- The proposed SCA is validated with the MFO and EP techniques.

The rest of this paper is organized as follows: In Section 2, a mathematical modelling of the SMIB system and the PSS damping controller parameters for improving damping capabilities of the controller are described. The formulation of the objection function for eigenvalue analysis is illustrated in Section 3. An overview of the optimization techniques is given in Section 4. The results under three loading conditions are presented in Section 5. Conclusions are presented in Section 6. 


\section{Mathematical Model of SMIB System with PSS}

Figure 1 shows a simple representation of the SMIB power system, which is used for stability purposes, in operation. Figure 2 shows a synchronous generator $G$, with excitation system, amortisseurs, terminal voltage $E_{t}$, and a PSS connected to an infinite bus with voltage $E_{B}$ through transmission lines of impedance $Z_{e q}$, with $R_{E}$ and $X_{E}$ as real and imaginary parts, respectively, that is used to design excitation and PSS damping controllers [1].

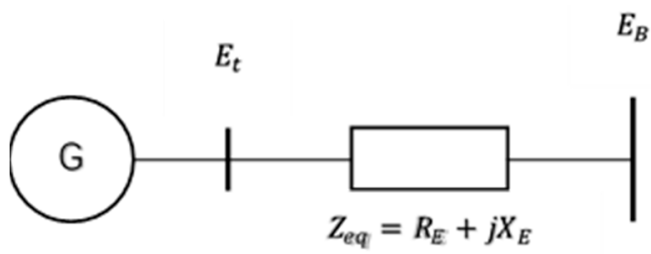

Infinite bus

Figure 1. Machine connected to a large system.

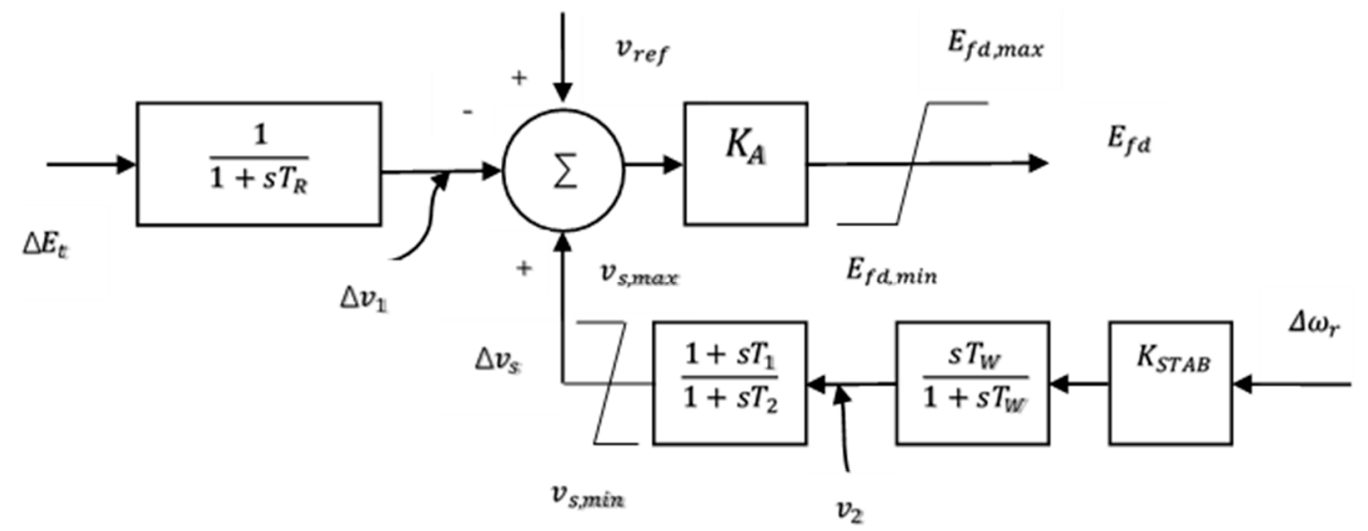

Figure 2. Excitation system with a PSS and AVR.

Then, by using a PSS with a basic structure model including AVR, $K_{A}$ is the exciter system gain constant and $T_{R}$ is the exciter system constant. $K_{S T A B}, T_{1}, T_{2}$, and $T_{W}$ are the gain, time constants for phase compensation, and washout of the PSS, respectively.

Figure 3 shows the block diagram of the SMIB with a PSS, AVR, and exciter. $H$ is the constant of inertia and $K_{D}$ is the damping torque coefficient. $K_{1}, K_{2}$, and $K_{4}$ are machine constants and $K_{3}, K_{5}$, and $K_{6}$ are the SMIB and excitation systems with PSS and AVR constants.

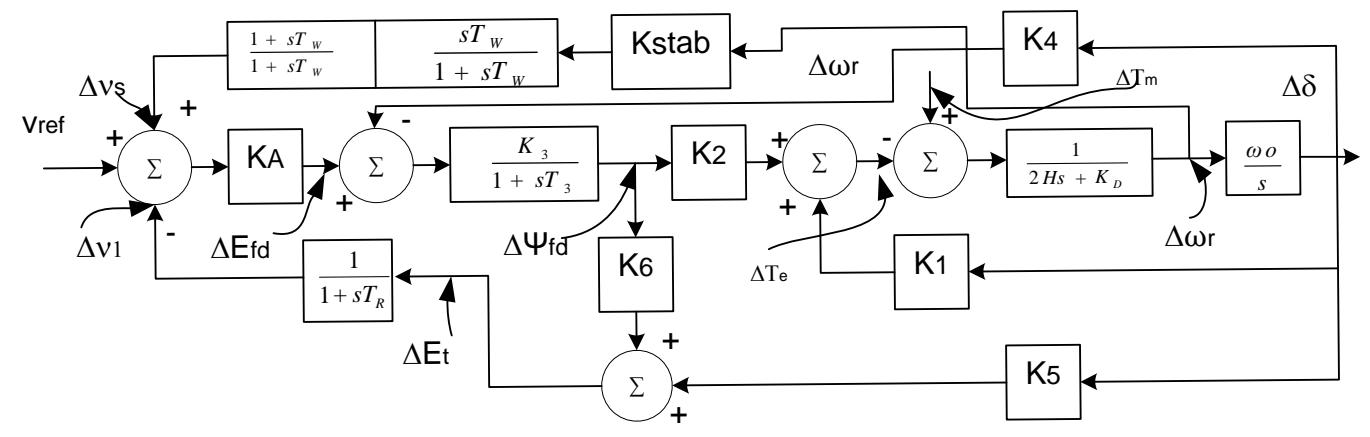

Figure 3. Block diagram the SMIB with a PSS, AVR, and exciter.

Referring to Figure 3, the equations representing the SMIB can be derived as follows:

$$
\Delta \dot{\omega}_{r}=-\frac{K_{D}}{2 H} \Delta \omega_{r}-\frac{K_{1}}{2 H} \Delta \delta-\frac{K_{2}}{2 H} \Delta \psi_{f d}-\frac{K_{21}}{2 H} \Delta \psi_{1 d}-\frac{K_{22}}{2 H} \Delta \psi_{1 q}-\frac{K_{23}}{2 H} \Delta \psi_{2 q}+\frac{1}{2 H} \Delta T_{m}
$$




$$
\begin{gathered}
\Delta \dot{\delta}=2 \pi f_{0} \Delta \omega_{r} \\
\Delta \dot{\psi}_{f d}=\mathrm{A} 32 \Delta \delta+\mathrm{A} 33 \Delta \psi_{f d}+\mathrm{A} 34 \Delta \psi_{1 d}+\mathrm{A} 35 \Delta \psi_{1 q}+\mathrm{A} 36 \Delta \psi_{2 q}+\mathrm{b} 32 \Delta \psi_{f d} \\
\Delta \dot{\psi}_{1 d}=\mathrm{A} 42 \Delta \delta+\mathrm{A} 43 \Delta \psi_{f d}+\mathrm{A} 44 \Delta \psi_{1 d}+\mathrm{A} 45 \Delta \psi_{1 q}+\mathrm{A} 46 \Delta \psi_{2 q} \\
\Delta \dot{\psi}_{1 q}=\mathrm{A} 52 \Delta \delta+\mathrm{A} 53 \Delta \psi_{f d}+\mathrm{A} 54 \Delta \psi_{1 d}+\mathrm{A} 55 \Delta \psi_{1 q}+\mathrm{A} 56 \Delta \psi_{2 q} \\
\Delta \dot{\psi}_{2 q}=\mathrm{A} 62 \Delta \delta+\mathrm{A} 63 \Delta \psi_{f d}+\mathrm{A} 64 \Delta \psi_{1 d}+\mathrm{A} 65 \Delta \psi_{1 q}+\mathrm{A} 66 \Delta \psi_{2 q}
\end{gathered}
$$

where $\delta$ is the rotor angle; $\omega$ is the rotor speed; $\psi_{f d}$ is the field flux; $\psi_{1 d}$ is the flux due to the d-axis amortisseur; $\psi_{1 q}$ is the flux due to the q-axis first amortisseur; and $\psi_{2 q}$ is the flux due to the q-axis second amortisseur. Referring to Figure 2, the equations representing the excitor with the AVR and PSS are as follows:

$$
\begin{gathered}
\Delta \dot{v}_{1}=\frac{K_{5}}{T_{R}} \Delta \delta+\frac{K_{6}}{T_{R}} \Delta \psi_{f d}+\frac{K_{61}}{T_{R}} \Delta \psi_{1 d}+\frac{K_{62}}{T_{R}} \Delta \psi_{1 q}+\frac{K_{63}}{T_{R}} \Delta \psi_{2 q}-\frac{1}{T_{R}} \Delta v_{1} \\
\Delta \dot{v}_{2}=K_{S T A B}\left(\Delta \dot{\omega}_{r}\right)-\frac{1}{T_{W}} \Delta v_{2} \\
\Delta \dot{v}_{s}=\frac{T_{1}}{T_{2}}\left(\Delta \dot{v}_{2}\right)+\frac{1}{T_{2}} \Delta v_{2}-\frac{1}{T_{2}} \Delta v_{s}
\end{gathered}
$$

The system in Figure 3 linearized with state space model is given by:

$$
\begin{gathered}
\dot{x}=\mathrm{Ax}+\mathrm{Bu} \\
\dot{x}=\left[\Delta \dot{\omega}_{r} \Delta \dot{\delta} \Delta \dot{\psi}_{f d} \Delta \dot{\psi}_{1 d} \Delta \dot{\psi}_{1 q} \Delta \dot{\psi}_{2 q} \Delta \dot{v}_{1} \Delta \dot{v}_{2} \Delta \dot{v}_{s}\right] \\
x=\left[\Delta \omega_{r} \Delta \delta \Delta \psi_{f d} \Delta \psi_{1 d} \Delta \psi_{1 q} \Delta v_{1} \Delta v_{2} \Delta v_{s}\right] \\
u=\left[\begin{array}{c}
\Delta T_{m} \\
\Delta E_{f d}
\end{array}\right]
\end{gathered}
$$

$A=\left[\begin{array}{ccccccccc}-\frac{K_{D}}{2 H} & -\frac{K_{1}}{2 H} & -\frac{K_{2}}{2 H} & -\frac{K_{21}}{2 H} & -\frac{K_{22}}{2 H} & -\frac{K_{23}}{2 H} & 0 & 0 & 0 \\ 2 \pi f_{0} & 0 & 0 & 0 & 0 & 0 & 0 & 0 & 0 \\ 0 & A_{32} & A_{33} & A_{34} & A_{35} & A_{36} & 0 & 0 & 0 \\ 0 & A_{42} & A_{43} & A_{44} & A_{45} & A_{46} & 0 & 0 & 0 \\ 0 & A_{52} & A_{53} & A_{54} & A_{55} & A_{56} & 0 & 0 & 0 \\ 0 & A_{62} & A_{63} & A_{64} & A_{65} & A_{66} & 0 & 0 & 0 \\ 0 & \frac{K_{5}}{T_{R}} & \frac{K_{6}}{T_{R}} & \frac{K_{61}}{T_{R}} & \frac{K_{62}}{T_{R}} & \frac{K_{63}}{T_{R}} & -\frac{1}{T_{R}} & 0 & 0 \\ -\frac{K_{D}}{2 H} K_{S T A B} & -\frac{K_{1}}{2 H} K_{S T A B} & -\frac{K_{2}}{2 H} K_{S T A B} & -\frac{K_{21}}{2 H} K_{S T A B} & -\frac{K_{22}}{2 H} K_{S T A B} & -\frac{K_{23}}{2 H} K_{S T A B} & 0 & -\frac{1}{T_{W}} & 0 \\ -\frac{T_{1}}{T_{2}} \frac{K_{D}}{2 H} K_{S T A B} & -\frac{T_{1}}{T_{2}} \frac{K_{1}}{2 H} K_{S T A B} & -\frac{T_{1}}{T_{2}} \frac{K_{2}}{2 H} K_{S T A B} & -\frac{T_{1}}{T_{2}} \frac{K_{21}}{2 H} K_{S T A B} & -\frac{T_{1}}{T_{2}} \frac{K_{22}}{2 H} K_{S T A B} & -\frac{T_{1}}{T_{2}} \frac{K_{23}}{2 H} K_{S T A B} & 0 & -\frac{T_{1}}{T_{2}} \frac{1}{T_{W}} & -\frac{1}{T_{2}}\end{array}\right]$

$$
\begin{gathered}
B=\left[\begin{array}{cc}
\frac{1}{2 H} & 0 \\
0 & 0 \\
0 & \frac{\omega_{0}}{l_{a d u}} R_{f d} \\
0 & 0 \\
0 & 0 \\
0 & 0 \\
0 & 0 \\
\frac{K_{S T A B}}{2 H} & 0 \\
\frac{T_{1}}{T_{2}} \frac{K_{S T A B}}{2 H} & 0
\end{array}\right] \\
\mathrm{A} 32=-\frac{\omega_{0} R_{f d} m_{1} \hat{l}_{a d s}}{l_{f d}}
\end{gathered}
$$




$$
\begin{aligned}
& \mathrm{A} 33=-\frac{\omega_{o} R_{f d}}{l_{f d}}\left(1-\frac{\hat{l}_{a d s}}{l_{f d}}+m_{2} \ddot{l}_{a d s}\right) \\
& \mathrm{A} 34=-\frac{\omega_{0} R_{f d}}{l_{f d}}\left(-\frac{\hat{l}_{a d s}}{l_{f d}}+m_{3} \ddot{l}_{a d s}\right) \\
& \mathrm{A} 35=-\frac{\omega_{0} R_{f d} m_{4} \ddot{l}_{a d s}}{l_{f d}} \\
& \mathrm{~A} 36=-\frac{\omega_{0} R_{f d} m_{5} \ddot{l}_{a d s}}{l_{f d}} \\
& \mathrm{~A} 42=-\frac{\omega_{0} R_{1 d} m_{1} \ddot{l}_{a d s}}{l_{1 d}} \\
& \mathrm{~A} 43=-\frac{\omega_{0} R_{1 d} \ddot{l}_{a d s}}{l_{1 d}}\left(m_{2}-\frac{1}{l_{f d}}\right) \\
& \mathrm{A} 44=-\frac{\omega_{0} R_{1 d}}{l_{1 d}}\left(\ddot{l}_{a d s} m_{3}-\frac{\ddot{l}_{a d s}}{l_{1 d}}+1\right) \\
& \mathrm{A} 45=-\frac{\omega_{0} R_{1 d} m_{4} \ddot{l}_{a d s}}{l_{1 d}} \\
& \mathrm{~A} 46=-\frac{\omega_{0} R_{1 d} m_{5} \ddot{l}_{a d s}}{l_{1 d}} \\
& \mathrm{~A} 52=-\frac{\omega_{0} R_{1 q} n_{1} \ddot{l}_{\text {aqs }}}{l_{1 q}} \\
& \mathrm{~A} 53=-\frac{\omega_{0} R_{1 q} n_{2} \ddot{l}_{\text {aqs }}}{l_{1 q}} \\
& \mathrm{~A} 54=-\frac{\omega_{0} R_{1 q} n_{3} \ddot{l}_{a q s}}{l_{1 q}} \\
& \mathrm{~A} 55=-\frac{\omega_{0} R_{1 q}}{l_{1 q}}\left(1-\frac{\ddot{l}_{a q s}}{l_{1 d}}+n_{4}\right) \\
& \mathrm{A} 56=-\frac{\omega_{0} R_{1 q} \ddot{l}_{\text {aqs }}}{l_{1 q}}\left(n_{5}-\frac{1}{l_{2 q}}\right) \\
& \mathrm{A} 62=-\frac{\omega_{0} R_{2 q} n_{1} \ddot{l}_{a q s}}{l_{2 q}} \\
& \mathrm{~A} 63=-\frac{\omega_{0} R_{2 q} n_{2} \ddot{l}_{\text {qqs }}}{l_{2 q}} \\
& \mathrm{~A} 64=-\frac{\omega_{0} R_{2 q} n_{3} \ddot{l}_{a q s}}{l_{2 q}} \\
& \mathrm{~A} 65=-\frac{\omega_{0} R_{2 q} \ddot{l}_{a q s}}{l_{2 q}}\left(n_{4}-\frac{1}{l_{1 q}}\right) \\
& \mathrm{A} 66=-\frac{\omega_{0} R_{2 q}}{l_{2 q}}\left(\ddot{l}_{a q s} n_{5}-\frac{\ddot{l}_{a q s}}{l_{2 q}}+1\right)
\end{aligned}
$$


$K_{21}, K_{22}$, and $K_{23}$ are machine constants and $K_{61}, K_{62}$, and $K_{63}$ are the SMIB and excitation system with PSS and AVR constants. The SMIB equations can be expressed in a $\mathrm{d}$-q refence frame of the machine. The currents in the d-axis and q-axis of the machine in terms of state variables $\psi_{f d}$ and $\delta$ and their perturbed values can be written as follows:

$$
\begin{gathered}
\Delta i_{d}=m_{1} \Delta \delta+m_{2} \Delta \psi_{f d} \\
\Delta i_{q}=n_{1} \Delta \delta+n_{2} \Delta \psi_{f d}
\end{gathered}
$$

where $m_{1}=E_{B}\left(X_{T q} \sin \delta_{o}-R_{T} \cos \delta_{o}\right) / \mathrm{D}, n_{1}=E_{B}\left(R_{T} \sin \delta_{o}-X_{T d} \cos \delta_{o}\right) / \mathrm{D}, m_{2}=\frac{X_{T q} L_{a d s}}{D\left(L_{a d s}+L_{f d}\right)}$, $n_{2}=\frac{R_{T} L_{a d s}}{D\left(L_{a d s}+L_{f d}\right)}, R_{T}=R_{a}+R_{E}, X_{T q}=X_{E}+X_{q s}, X_{T d}=X_{E}+\dot{X}_{d s}$, and $\mathrm{D}=R_{T}^{2}+X_{T q} X_{T d}$.

The perturbed values of electrical torque and field current are obtained from the perturbed values currents $\Delta i_{d}$ and $\Delta i_{q} . L_{a d s}$ and $L_{a q s}$ are saturated values of mutual inductances. The change in mechanical torque is zero with constant mechanical input torque.

$$
\Delta T_{e}=K_{1} \Delta \delta+K_{2} \Delta \psi_{f d}
$$

where $K_{1}=n_{1}\left(\psi_{a d o}+L_{a q s} i_{d o}\right)-m_{1}\left(\psi_{a q o}+i_{a d s} i_{q o}\right)$ and $K_{2}=n_{2}\left(\psi_{a d o}+L_{a q s} i_{d o}\right)-m_{2}\left(\psi_{a q o}+\right.$ $\left.i_{a d s} i_{q o}\right)+\frac{i_{a d s}}{L_{f d}} i_{q o}$.

In the small signal performance analysis, a difference is noted between saturation and incremental saturation for perturbed values of currents and flux linkages. The term saturation is related to total flux linkages and current and incremental saturation is related to the perturbed values of currents and flux linkages. The expressions for $K_{3}$ and $K_{4}$ are as follows:

$$
\begin{gathered}
K_{3} \frac{L_{a d s}+L_{f d}}{L_{a d u}\left(1+\left(X_{d}-\dot{X}_{d}\right) \frac{X_{T q}}{D}\right)} \\
K_{4} \frac{m_{1} L_{a d s}}{L_{a d s}+L_{f d}}
\end{gathered}
$$

The effect of field flux on the system stability with a constant field voltage and a perturbed $\Delta \psi_{f d}$ is zero, so the field flux changes due to feedback of $\Delta \delta$ through $K_{4}$, showing a demagnetizing effect of the armature reaction. The rotor angle variations with changes in air gap torque due to field flux changes are given as follows:

$$
\frac{\Delta T_{e}}{\Delta \delta}=-\frac{K_{2} K_{3} K_{4}}{1+s T_{3}}
$$

The perturbed linkage field flux, $\Delta \psi_{f d}$, affects the damping torque and synchronizing torque components and depends on the oscillating frequency as follows:

- Operating in a steady state and having very low oscillating frequencies. The armature reaction introduces a negative synchronizing torque, and when its value exceeds $K_{1} \Delta \delta$, the system become monotonically unstable and the steady state stability limit is reached at $K_{1}=K_{2} K_{3} K_{4}$.

- Operating at much higher oscillating frequencies than $\frac{1}{T_{3}}$. The component of the air gap torque due to $\Delta \psi_{f d}$ is in phase with $\Delta \omega$ and leads by $90^{\circ}$ from $\Delta \delta . \Delta \psi_{f d}$ introduces a positive damping torque component.

- Operating at a $1 \mathrm{~Hz}$ oscillating frequency. $\Delta \psi_{f d}$ introduces a negative synchronizing torque component and positive damping torque component. The collective effect is to increase the damping torque and reduce the synchronizing torque. When $K_{4}$ remains positive, $\Delta \psi_{f d}$ introduces a positive damping torque component due to the armature reaction. $K_{4}$ is negative on two conditions: (1) when the hydraulic generator without damper windings is operating at a light load and connected with high resistance to the reactance ratio line of a large system or (2) when the hydraulic generator is partly supplied by a large, local, connected load and partly supplied by a remote generator 
set. In this condition, induced torques are out of phase with $\Delta \omega$ and yield a negative damping.

Next, we outline the effect of an excitation system on the damping torque and synchronizing components. Referring to the block diagram in Figure 3, the equations of $\Delta \psi_{f d}$ with the AVR and change in air gap torque due to $\Delta \psi_{f d}$ are as follows:

$$
\begin{gathered}
\Delta \psi_{f d}=-\frac{K_{3}\left\{\left(1+s T_{R}\right)+K_{5} K_{A}\right\}}{s^{2} T_{3} T_{R}+s\left(T_{3}+T_{R}\right)+1+K_{3} K_{6} K_{A}} \Delta \delta \\
\Delta T_{e}=K_{2} \Delta \psi_{f d}
\end{gathered}
$$

With the AVR action, the effects of the perturbed linkage field flux $\Delta \psi_{f d}$, the damping torque, and synchronizing torque components depend on constant $K_{5}$ (negative) and oscillating frequency.

- Steady state synchronizing torque coefficient.

$$
\Delta T_{e}=-\frac{K_{2} K_{3}\left(K_{4}+K_{5} K_{A}\right)}{1+K_{3} K_{6} K_{A}} \Delta \delta
$$

The effect of the AVR is to increase the synchronizing torque components at a steady state by compensating for the effect of the armature reaction. When $K_{5}$ is positive, the AVR reduces the steady state synchronizing torque component.

- The damping torque and synchronizing torque components at oscillating frequency. When $K_{5}$ is negative for high values of the external system reactance and high generator outputs, the AVR increases the synchronizing torque component and reduces the damping torque component. The net synchronizing torque and damping torque are given as follows:

$$
\begin{gathered}
K_{S}=K_{1}+K_{s\left(\Delta \psi_{f d}\right)} \\
K_{D}=K_{D\left(\Delta \psi_{f d}\right)}
\end{gathered}
$$

With $K_{A}$ equal to zero, the only source of $\Delta \psi_{f d}$ is the armature reaction. $K_{D}$ reduces the increase of $K_{A}$. The net damping is zero and $\Delta \psi_{f d}$ is in phase with $\Delta \delta$ when $K_{A}$ is infinite. When $K_{5}$ is positive for low generator output and low values of external system reactance, the effect of the excitation system is to introduce a positive damping torque and negative synchronizing component torque. When $K_{1}$ has a high value, the net $K_{S}$ is greater than zero. However, the exciter introduces negative damping. The design of the exciter controller with a high response is meant to provide a compromise that results in sufficient damping and synchronizing torque components in the expected range of system operating conditions. An effective method to meet the incompatible exciter performance requirements regarding system stability is the use of a power system stabilizer (PSS).

The PSS produces a component air gap torque in phase with the rotor speed deviation; see the block diagram machine with excitation and a PSS shown in Figures 2 and 3. The PSS provides pure damping torque at all oscillating frequencies in a system by phase compensation for the phase lag between the air gap torque and exciter input. The $\Delta \psi_{f d}$ due to the PSS, by neglecting $T_{R}$, is given as follows:

$$
\frac{\Delta \psi_{f d}}{\Delta v_{s}}=\frac{K_{3} K_{A}}{s T_{3}+1+K_{3} K_{6} K_{A}}
$$

The PSS phase compensation introduces damping torque at any rotor oscillating frequency, $K_{S T A B}$ determines the amount of damping required, and the washout block decides the PSS responses only to the change in rotor speed. The PSS ensures the overall system stability.

The effect of amortisseurs is to increase the damping ratio with a slight increase in frequency. This increase in frequency indicates an increment in synchronizing torque. 
The effect of AVR is to introduce negative damping. The system is unstable for all higher values of $K_{A}$ (above 14). The damping of the rotor angle mode increases by increasing the PSS gain and slightly decreasing in synchronizing torque $[1,30]$. Comprehensive calculation of the system in Figure 3 and values of perturbed terminal voltage, air gap torque, transfer function of exciter with the AVR, field and mutual flux linkages neglecting stator transients, current variations in d-axis and q-axis, internal rotor angle, and saturation factor values for a synchronous generator can found in references [1,30].

\section{Analysis of Eigenvalues}

This research assesses the angle stability using eigenvalue analysis. Eigenvalues with more negative than positive signs represent a more stable system. An eigenvalue consists of real and imaginary parts, which have both oscillatory and exponential decay modes if the system is stable. Exponential decay corresponds to a negative real value of an eigenvalue and an imaginary value of the eigenvalue corresponds to an oscillatory nature. The conjugate complex pair of eigenvalues represents the oscillatory mode. The imaginary part of the conjugate complex eigenvalue represents the frequency of oscillation in the power system. The damping ratio is the ratio between the negative real value of a complex eigenvalue to the square root of the sum of the square of the real and imaginary values of the complex eigenvalues. Any change in damping ratio affects the power system stability. Specifically, the power system stability increases as the damping ratio increases and shifts towards larger negative real eigenvalues in the left half of the complex s-plane. The eigenvalues can be derived from the linearized state space model matrix (A) of the system by using Equation (36):

$$
\lambda_{j}=\operatorname{eig}(\mathrm{A})
$$

where $j=1,2,3 \ldots \mathrm{n}, \mathrm{n}$ is equal to state variables in the system and eig is a built-in function in MATLAB. The objective function formulated to minimize the damping factor is Minimize;

$$
\mathrm{f}=\max \left\{\operatorname{real}\left(\lambda_{j}\right)\right\}
$$

Three parameters of the PSS lead lag, $T_{1}, T_{2}$, and $T_{W}$, need optimization subject to the following power system stabilizer parameters:

$T_{1}$,pass,min $\leq T_{1}$,pass $\leq T_{1}$,pass, $\max$

$T_{2}$,pass, $\min \leq T_{2}$,pass $\leq T_{2}$,pass, $\max$

$T_{W}$,pass, $\min \leq T_{W}$,pass $\leq T_{W}$,pass, $\max$

\section{Metaheuristic Optimization Techniques}

Heuristic optimization algorithms are nature inspired and find the solution through trial and error by using stochastic methods. Metaheuristic algorithms are the improved version of heuristic algorithms; they are developed on the basis nature-inspired events. These optimization techniques are more flexible, robust, and do not need to predict the initial solution. The optimization techniques implemented in this paper are outlined in this section [4]:

\subsection{Overview of the SCA Algorithm}

This SCA is a nature-inspired stochastic population-based optimization metaheuristic technique developed by Mirjalili; sine and cosine functions in mathematics find applications in nature [31]. SCA initializes a search randomly and each candidate solution is updated using Equation (38):

$$
X_{i, j}^{t+1}=\left\{\begin{array}{l}
X_{i, t}+r_{1}^{t} \sin r_{2, j}\left|r_{3, j} X_{d, j}^{t}-X_{i, j}^{t}\right|, \text { if } r_{4, j}<0.5 \\
X_{j, t}+r_{1}^{t} \cos r_{2, j}\left|r_{3, j} X_{d, j}^{t}-X_{i, j}^{t}\right|, \text { if } r_{4, j} \geq 0.5
\end{array}\right.
$$


where $X_{i, t}$ and $X_{i, t+1}$ represent the $i^{t h}$ solution vector at $t^{\text {th }}$ and $(t+1)^{t h}$ solution at the $(t+1)$ iteration, respectively; $X_{d, j}^{t}$ is the best solution in each iteration; $r_{4, j}$ is a random number in the range $0-1 ; r_{2, j}$ is a random number between 0 and $2 \pi$, deciding the direction of the solution either towards or away from the current one; $r_{1}^{t}$ is the random number that defines control of the exploration and exploitation in the search using the equation $r_{1}^{t}=2-2 \times\left(\frac{t}{t_{\max }}\right)$, where $t_{\max }$ is the maximum number of iterations that a user needs; $t$ is the current generation number; $r_{1}^{t}$ provides exploration for the first half of the iterations and exploitations for the second half; $r_{3, j}$ is the random number that provides the weight to $X_{d, j}^{t}$ to find the best solution by needful exploration and exploitation in a random search space. Figure 4 shows the flowchart of the SCA.

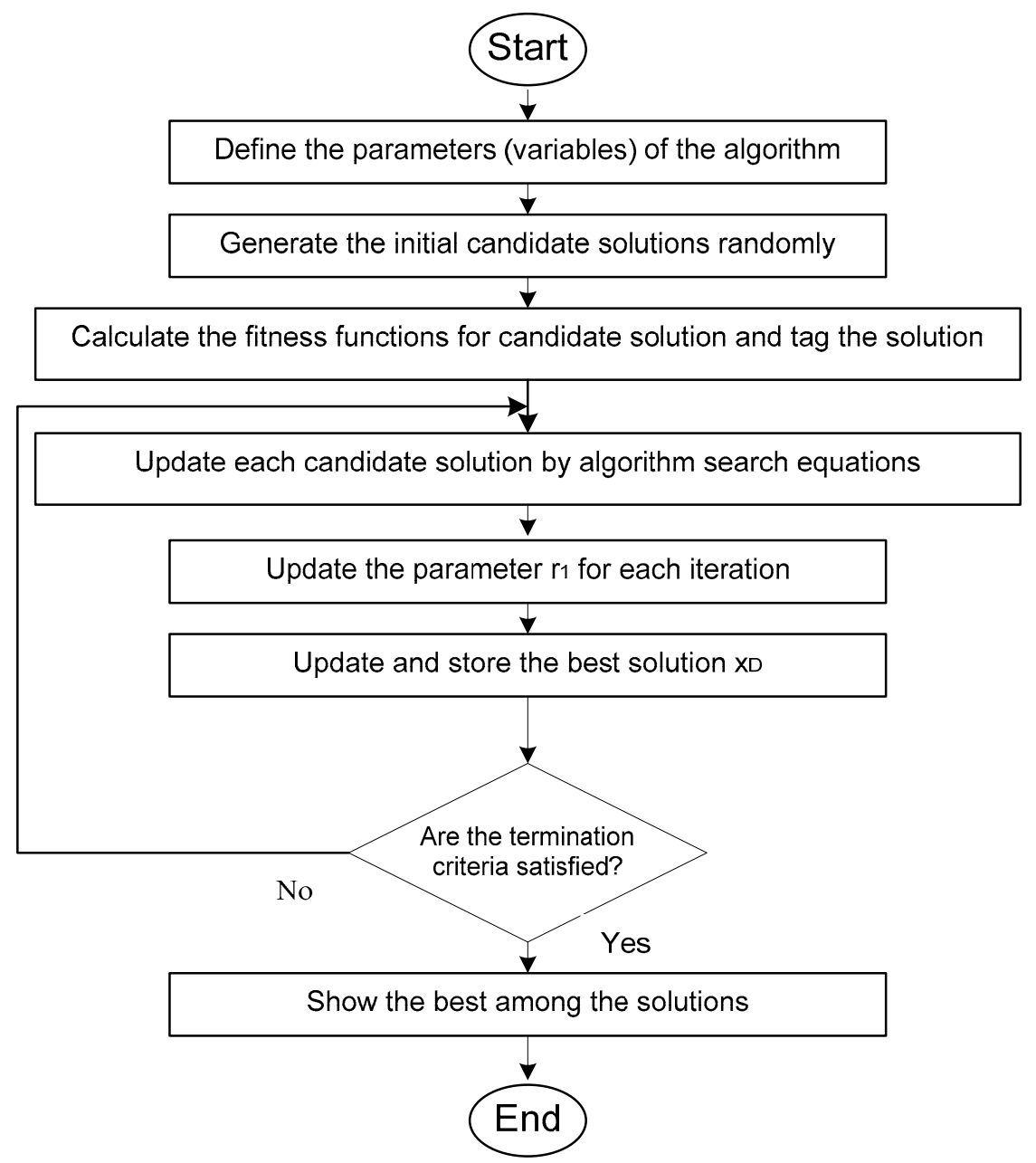

Figure 4. Flow chart of the SCA.

\subsection{Overview of the MFO Algorithm}

Moths are insects similar to butterflies and have special navigation methods while moving at night using moonlight. Moths move towards the destination by adapting a mechanism called transverse orientation for navigation, maintaining a fixed angle with the light source and taking a spiral path around and towards the light source. Moths' flight behavior was modelled mathematically to propose the MFO algorithm. MFO is metaheuristic; it starts by defining parameters and then generates moths randomly, calculates the position of each moth, and tags the best solution with respect to the light source (flame). The next positions of the moths are updated depending on the spiral path function to obtain better positions with respect to the light source. This process, updating next positions and tagging the best solutions with respect to flame, is repeated and terminated when the criteria are 
satisfied (after obtaining the best solution). Figure 5 shows the flowchart of the MFO algorithm [32].

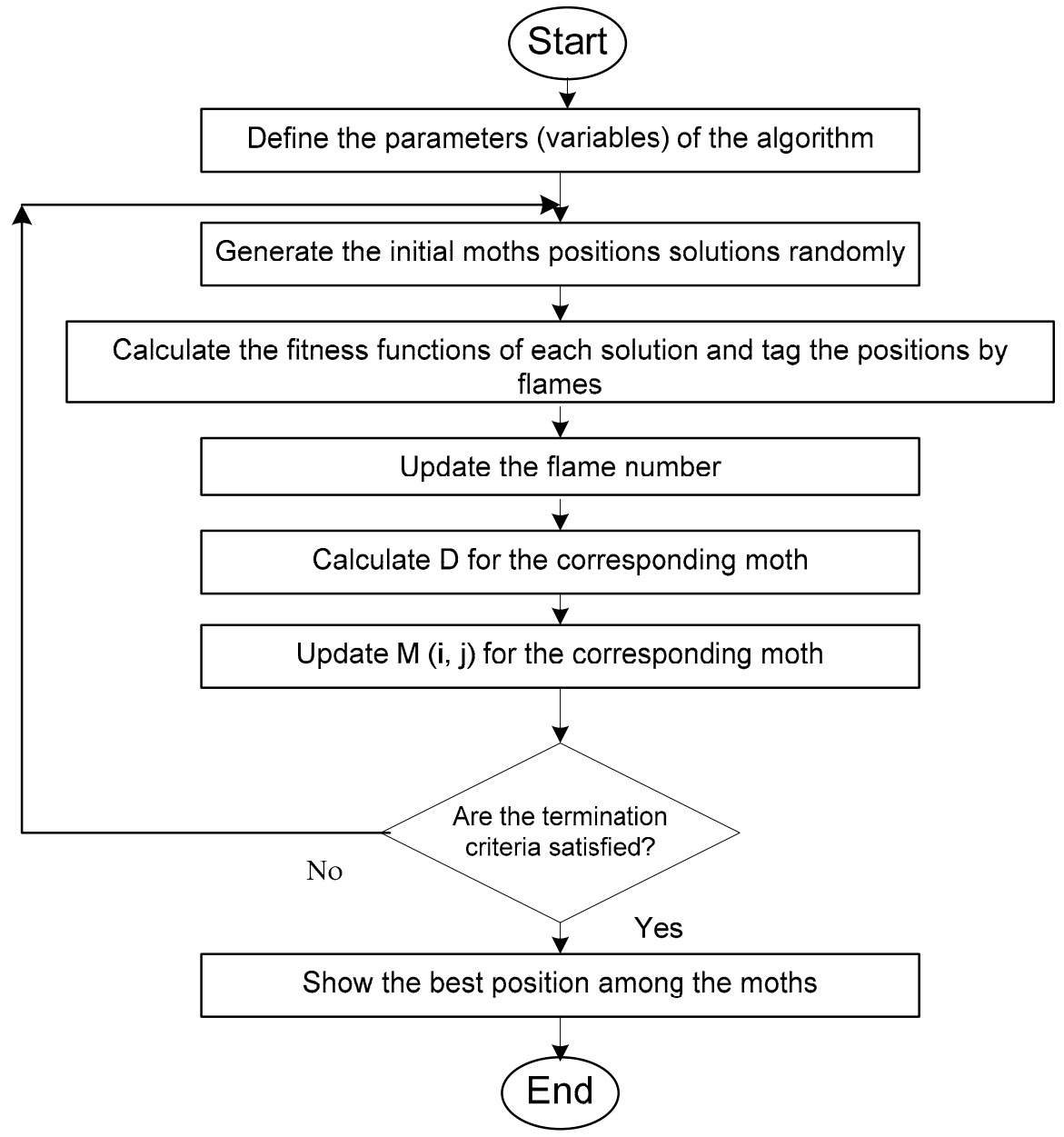

Figure 5. Flow chart of the MFO.

MFO has three main steps. First, the initial population of moths is generated. Second, the moth positions are updated. Third, the number of flames is updated.

In the first step, generating the initial population of moths assumes that each moth can move in multidimensional space. The set of moths in matrix form is:

$$
M=\left[\begin{array}{cccc}
M_{11} & M_{12} & \cdots & M_{1 d} \\
M_{21} & M_{22} & \cdots & M_{2 d} \\
\vdots & \vdots & \vdots & \vdots \\
M_{n 1} & M_{n 2} & \cdots & M_{n d}
\end{array}\right]
$$

where $n$ represents the number of moths and $d$ represents the dimension in space. For all moths, the fitness values are stored in an array.

$$
O M=\left[\begin{array}{c}
O M_{1} \\
O M_{2} \\
\vdots \\
O M_{n}
\end{array}\right]
$$


The matrix for flames in d-dimensional space is:

$$
F=\left[\begin{array}{cccc}
F_{11} & F_{12} & \cdots & F_{1 d} \\
F_{21} & F_{22} & \cdots & F_{2 d} \\
\vdots & \vdots & \vdots & \vdots \\
F_{n 1} & F_{n 2} & \cdots & F_{n d}
\end{array}\right]
$$

For all flames, the fitness values are stored in an array.

$$
O F=\left[\begin{array}{c}
O F_{1} \\
O F_{2} \\
\vdots \\
O F_{n}
\end{array}\right]
$$

Second, the moth positions are updated using three functions to converge the global optimal,

$$
M F O=(I, P, T)
$$

where $I$ is the initial location of the moth in the search space, $P$ is the motion of the moth in the space, and $T$ is the termination of the search. The following equation represents function $I$.

$$
M(i, j)=(u b(i)-l b(j)) * \text { rand }()+l b(i)
$$

where $u b$ and $l b$ are the upper and lower bounds of the variable, respectively. The MFO algorithm logarithmic spiral equation guarantees the exploration and exploitation of the moth near the flame in the search space, as:

$$
S\left(M_{i}, F_{j}\right)=D_{i} \cdot e^{b t} \cdot \cos (2 \pi t)+F_{j}
$$

where $D_{i}$ is the distance between the $i$-th moth and $j$-th flame, $b$ is the spiral shape, and $t$ is a number between -1 and 1 . Third, the number of flames is updated, highlighting the exploitation of the MFO algorithm by decreasing the number of flames to overcome the issue.

$$
\text { Flame no }=\text { round }\left(N-l \frac{N-l}{T}\right)
$$

where $N$ is the maximum number of flames, $l$ is the current iteration number, and $T$ is the maximum number of iterations.

\subsection{Evolutionary Programming Algorithm}

The EP technique is based on biological evolution to find an optimal solution in a search space for complex engineering problems. The EP algorithm has six main steps: initialization by generation of a random solution; mutation using a Gaussian random variable; calculation of the maximum, minimum, and average fitness; determination of the best solution and combinations; selection of a higher candidate for the next generation; and termination of the process after the criteria are satisfied. Figure 6 shows the flow chart of the EP algorithm [30]. 


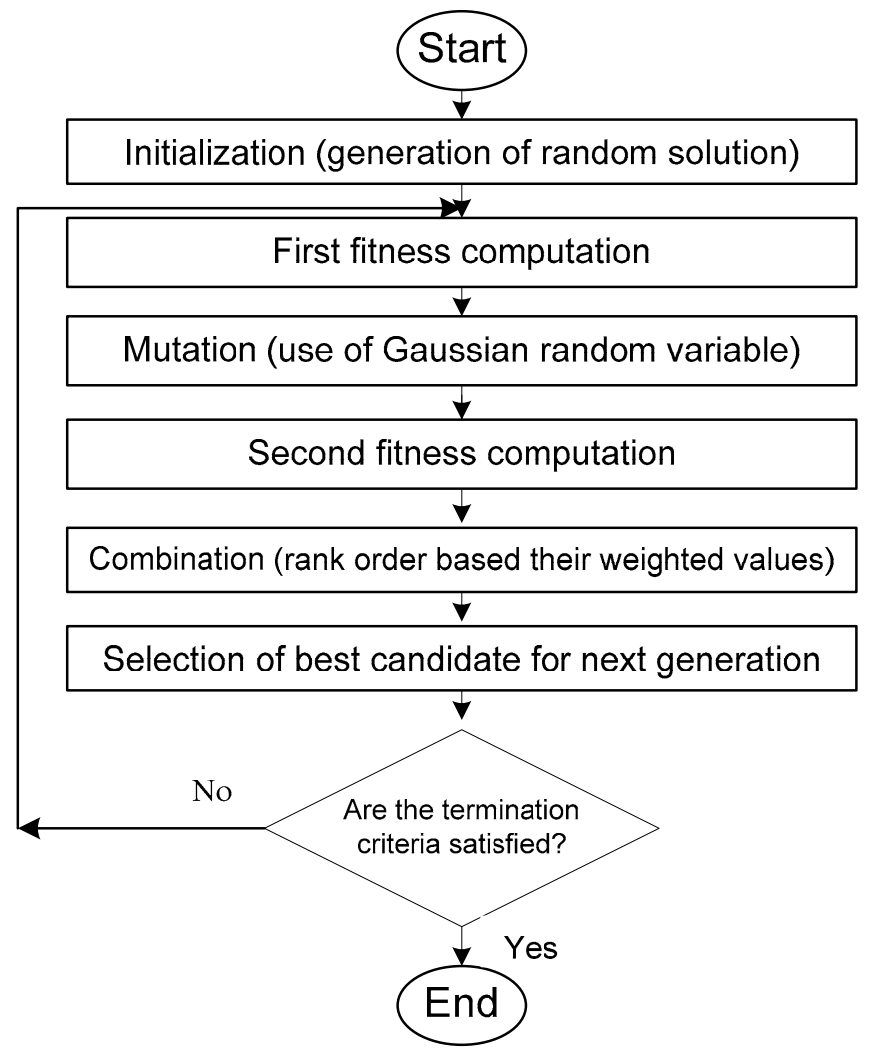

Figure 6. Flow chart of the EP.

\section{Results and Discussion}

In this study, simulations of a SMIB power system using SCA, MFO and EP techniques were carried out in MATLAB. Table 1 shows the three PSS parameters $T_{1}, T_{2}$, and $T_{W}$ under three different loading conditions, optimized until the minimum value of objective function was obtained.

Table 1. Cases with the loading conditions.

\begin{tabular}{ccc}
\hline \multirow{2}{*}{ Cases } & \multicolumn{2}{c}{ Loading Condition $(\mathbf{P}, \mathbf{Q})$} \\
\cline { 2 - 3 } & Active Load, $\mathbf{P}(\mathbf{p} . \mathbf{u})$ & Reactive Load, $\mathbf{Q}(\mathbf{p} . \mathbf{u})$ \\
\hline case 1 & $\mathrm{P}=0.9$ & $\mathrm{Q}=0.3$ \\
case 2 & $\mathrm{P}=0.5$ & $\mathrm{Q}=0.5$ \\
case 3 & $\mathrm{P}=0.2$ & $\mathrm{Q}=0.7$ \\
\hline
\end{tabular}

Four different approaches are discussed as follows:

1. SMIB system with an unoptimized PSS (PSSU)

2. SMIB system with a PSS optimized by the sine cosine algorithm (PSS-SCA)

3. SMIB system with a PSS optimized by the moth flame optimization (PSS-MFO)

4. SMIB system with a PSS optimized by evolutionary programming (PSS-EP)

Table 2 shows the generator, transmission line, exciter, and PSS parameters, where $\mathrm{H}$ is the constant of inertia; Et is the terminal voltage; $\mathrm{X}_{\mathrm{d}}, \mathrm{R}_{\mathrm{a}}, \mathrm{X}_{\mathrm{d}}, \mathrm{X}_{\mathrm{q}}$, and $\mathrm{T}_{\mathrm{d} 0}$ ' are, respectively, the transient reactance, armature resistance, $\mathrm{d}$-axis reactance, q-axis reactance, and the circuit field time constant of the generator; Ksd and Ksq are synchronizing torque coefficients in the d-axis and q-axis of the generator field, respectively; Re and Xe are resistance and reactance of the transmission line, respectively; $\mathrm{X}_{\mathrm{L}}$ is load reactance; $K_{A}$ and $T_{R}$ are exciter gain and time constants, respectively; and $K_{S T A B}$ is a gain constant of the PSS. 
Table 2. Parameters for the SMIB and the PSS.

\begin{tabular}{cc}
\hline Components & List of Parameters \\
\hline Generator & $\mathrm{H}=3.5, E_{t}=1<36^{\circ}, \mathrm{X}_{\mathrm{d}}{ }^{\prime}=0.3, \mathrm{R}_{\mathrm{a}}=0.003$, \\
& $\mathrm{X}_{\mathrm{d}}=1.81, \mathrm{X}_{\mathrm{q}}=1.76, \mathrm{~T}_{\mathrm{d} 0}{ }^{\prime}=8, \mathrm{Ksd}=0.8491$, \\
Transmission line & $\mathrm{K}_{\mathrm{sq}}=0.8491$. \\
Exciter and PSS & $\mathrm{R}_{\mathrm{e}}=0, \mathrm{X}_{\mathrm{e}}=0.65, \mathrm{X}_{\mathrm{L}}=0.16$ \\
& $\mathrm{~K}_{A}=200, \mathrm{~T}_{R}=0.02, \mathrm{~K}_{S T A B}$ \\
\hline
\end{tabular}

Figure 7 shows the responses of the rotor angle deviation in the phase plane comparing PSS-SCA, PSS-MFO, PSS-EP, and PSS-U for case 1. The PSS-SCA system had the shortest damping time, approximately $1.8 \mathrm{~s}$, and the smallest oscillation rate and damping performance of the SMIB system compared with those of the other approaches. PSS-MFO and PSS-EP followed with damping before $1.9 \mathrm{~s}$ and then PSS-U with damping before $5 \mathrm{~s}$. The PSS-EP system required the fewest iterations (20-30) to converge on the minimum value objective function compared with those needed by the other approaches. The PSS-SCA and PSS-MFO systems required 75-150 iterations to converge on the solutions. The proposed method provided the maximum value of the objective function with only a little constraint to computational efficiency.

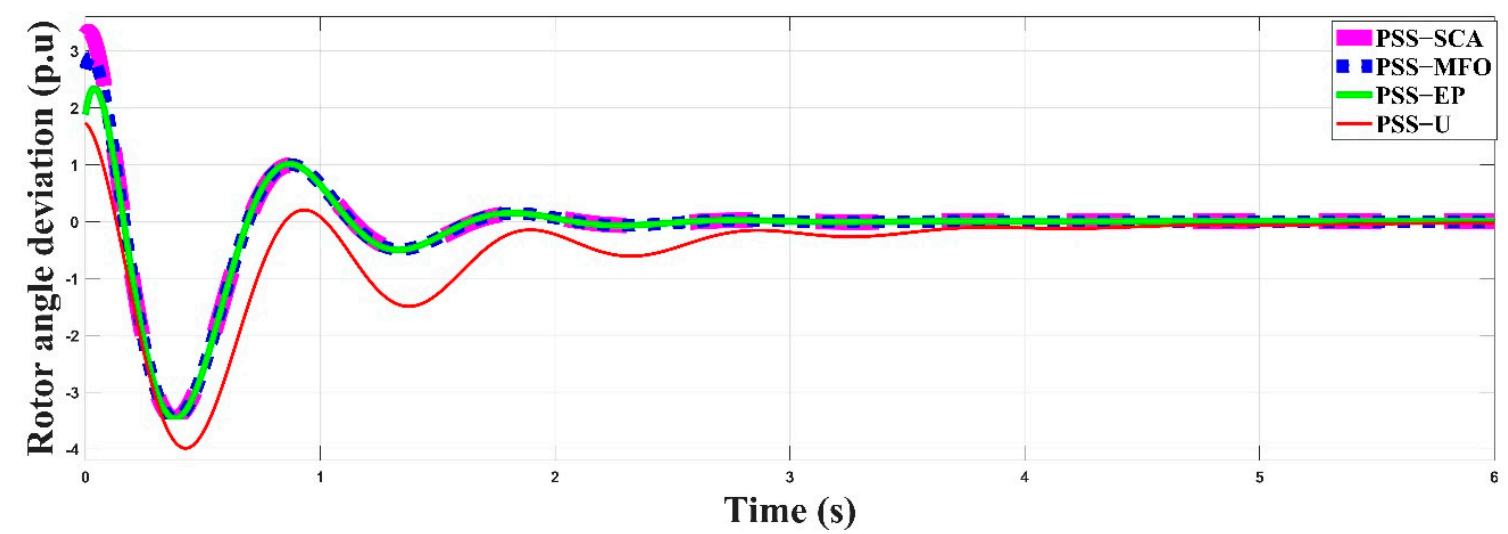

Figure 7. Rotor angle deviation response of PSSSCA, PSSMFO, PSS-EP, and PSS-U for case 1.

All eigenvalues are on the negative side of the complex s-plane, showing the stable condition of all systems for case 1, as shown in Figure 8. The eigenvalues of PSS-SCA are on the left-most of the imaginary axis of the s-plane and are shifted towards the real axis of the s-plane compared with those of the other techniques.

Table 3 shows the PSS parameters, $T_{1}, T_{2}$ and $T_{\mathrm{W}}$, of the lead lag controller tuned by the three optimization techniques with a minimum objective function for case 1 . This shows that the proposed PSS-SCA technique can tune the PSS parameters with a minimum objective function and stabilize the signal in a small system. 


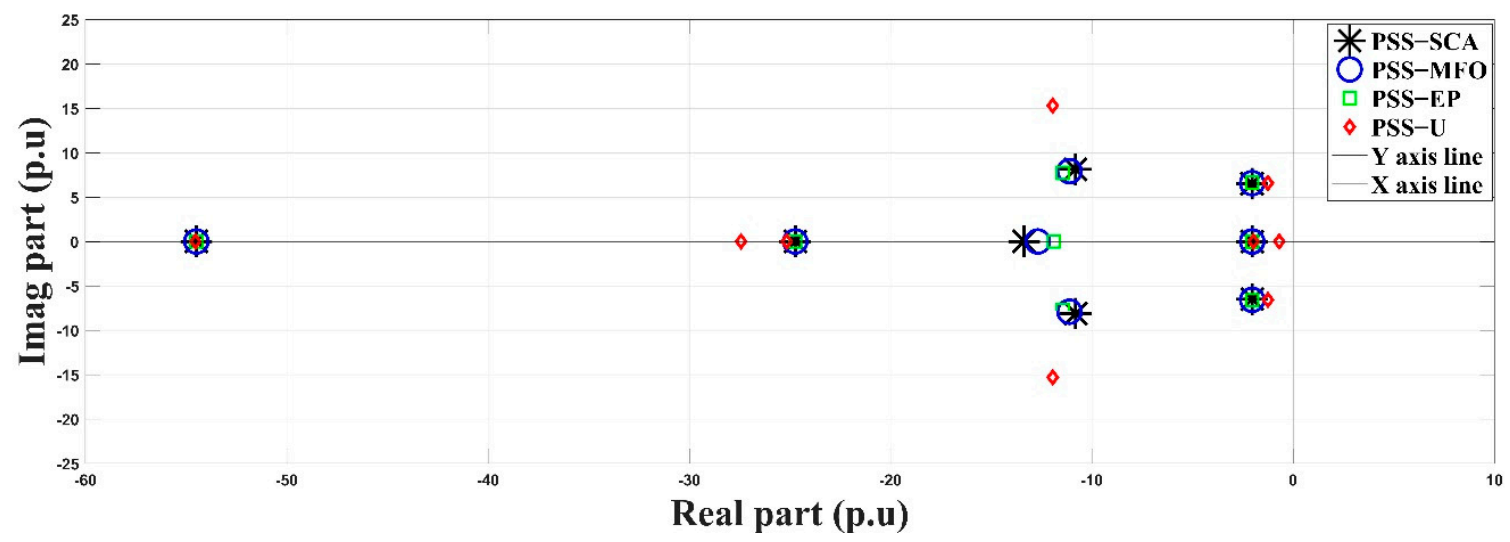

Figure 8. Eigenvalues distribution of PSS-SCA, PSS-MFO, PSS-EP, and PSS-U for case 1.

Table 3. Comparison of PSS-SCA, PSS-MFO, PSS-EP, and PSS-U for case 1.

\begin{tabular}{ccccc}
\hline Type & $\boldsymbol{T}_{\boldsymbol{w}}$ & $\boldsymbol{T}_{1}$ & $\boldsymbol{T}_{2}$ & Objective Function \\
\hline PSS-SCA & 0.4884 & 0.4327 & 0.2087 & -2.0298 \\
PSS-MFO & 0.4886 & 0.4330 & 0.2122 & -2.0294 \\
PSS-EP & 0.4886 & 0.4351 & 0.2167 & -2.0292 \\
PSS-U & 1.5 & 0.2 & 0.05 & -0.6881 \\
\hline
\end{tabular}

Figure 9 shows the responses of rotor angle deviation in the phase plane comparing PSS-SCA, PSS-MFO, PSS-EP, and PSS-U for case 2. The PSS-SCA had the shortest damping time, approximately $2.6 \mathrm{~s}$ and the smallest oscillation rate and damping performance of the SMIB system compared with those of the other approaches. PSS-MFO and PSS-EP showed damping before $2.7 \mathrm{~s}$ and PSS-U before $6 \mathrm{~s}$. The PSS-EP system required the fewest iterations (18-24) to converge on the minimum value objective function compared with those needed by the other approaches. The PSS-SCA and PSS-MFO systems required 52-130 iterations to converge on the solutions. The proposed method provided a maximum value of the objective function with only a little constraint to computational efficiency.

All eigenvalues are on the negative side of the complex s-plane, showing the stable condition of all systems for case 2, as shown in Figure 10. The eigenvalue of PSS-SCA is on the left-most of the imaginary axis and is shifted towards the real axis of the s-plane as compared with those of other techniques such as PSS-MFO, PSS-EP, and PSS-U.

Table 4 shows the PSS parameters, $T_{1}, T_{2}$ and $T_{W}$, of the lead lag controller tuned by the three optimization techniques with the minimum objective function for case 2 .

Figure 11 shows the responses of the rotor angle deviation in phase plane comparing PSS-SCA, PSS-MFO, PSS-EP, and PSS-U for case 3. The PSS-SCA system had the shortest damping time, approximately $2.9 \mathrm{~s}$, and the smallest oscillation rate and damping performance of the SMIB system compared with those of the other approaches. PSS-MFO and PSS-EP followed with damping before $3 \mathrm{~s}$ and then PSS-U with damping before $10 \mathrm{~s}$. The PSS-EP system required the fewest iterations (16-25) to converge on the minimum value objective function compared with those of the other approaches. The PSS-SCA and PSS-MFO systems required 60-130 iterations to converge on the solutions. The proposed method provided the maximum value of objective function with only a little constraint to computational efficiency. 

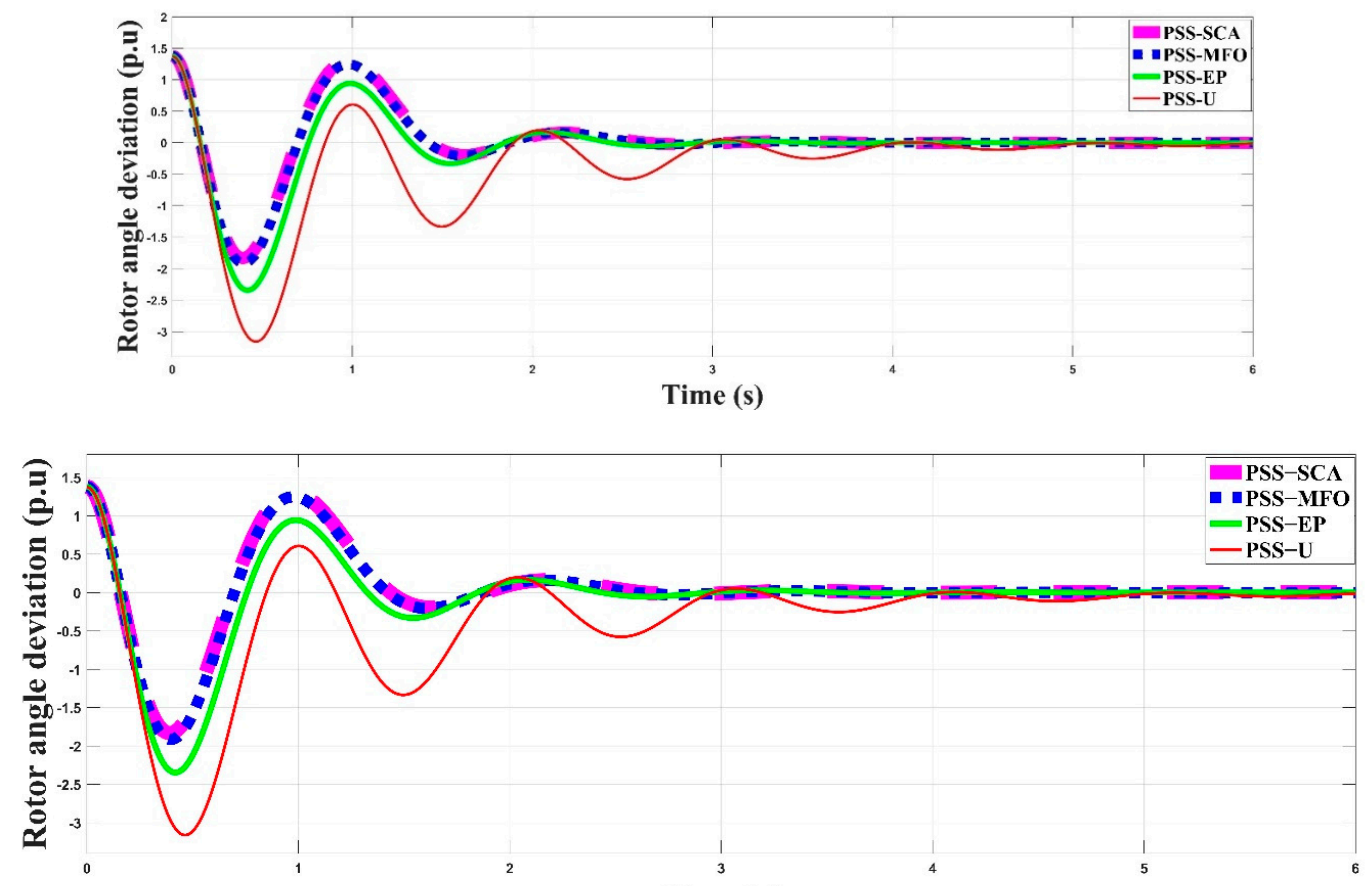

Time (s)

Figure 9. Rotor angle deviation response of PSS-SCA, PSS-MFO, PSS-EP, and PSS-U for case 2.
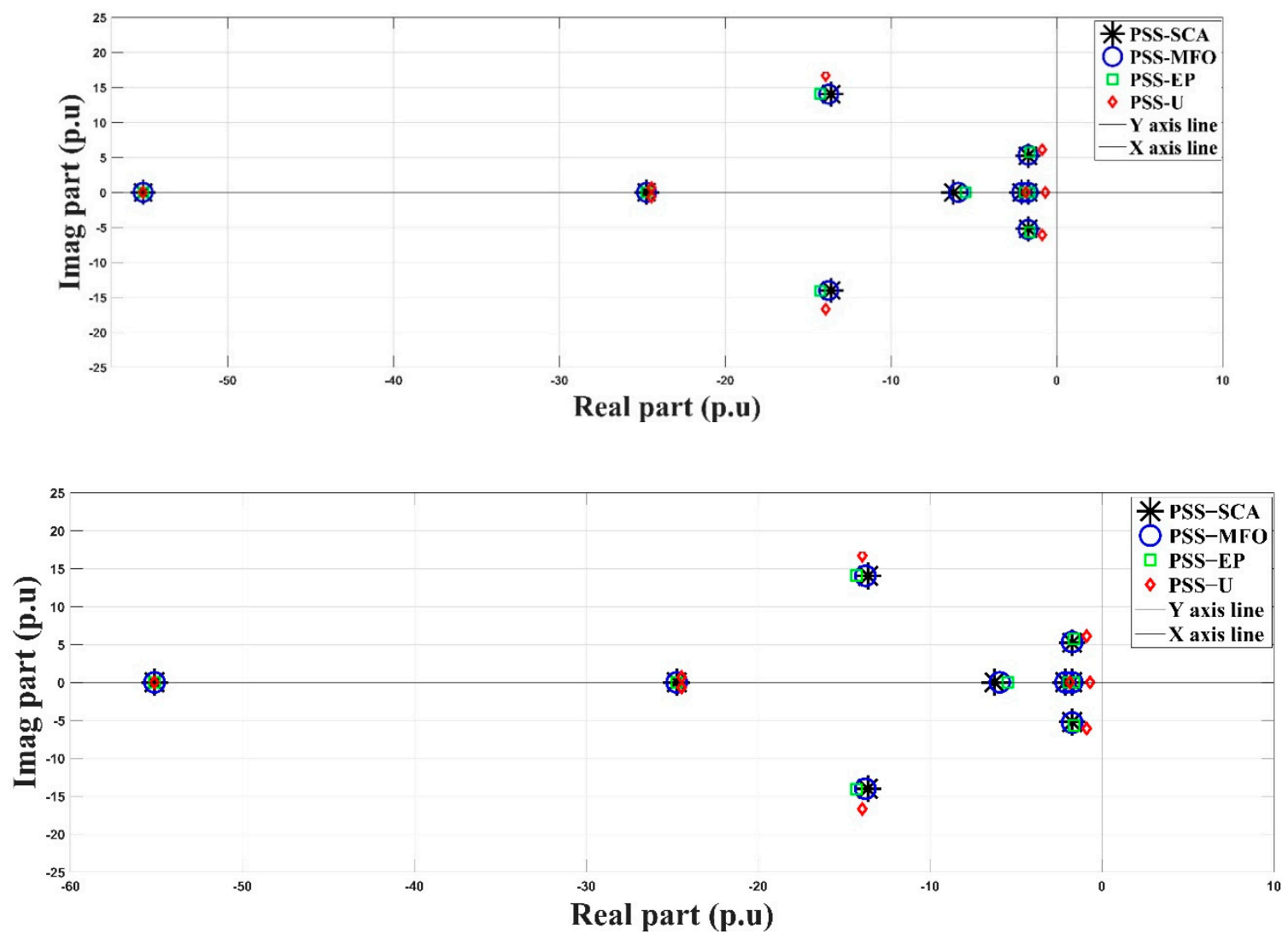

Figure 10. Eigenvalues distribution of PSS-SCA, PSS-MFO, PSS-EP, and PSS-U for case 2. 
Table 4. Comparison of PSS-SCA, PSS-MFO, PSS-EP, and PSS-U for case 2.

\begin{tabular}{ccccc}
\hline Type & $\boldsymbol{T}_{\boldsymbol{w}}$ & $\boldsymbol{T}_{1}$ & $\boldsymbol{T}_{2}$ & Objective Function \\
\hline PSS-SCA & 0.4324 & 1.1595 & 0.3583 & -1.7267 \\
PSS-MFO & 0.4472 & 1.1022 & 0.3513 & -1.7262 \\
PSS-EP & 0.5868 & 0.7875 & 0.2826 & -1.6269 \\
PSS-U & 1.5 & 0.2 & 0.05 & -0.6812 \\
\hline
\end{tabular}

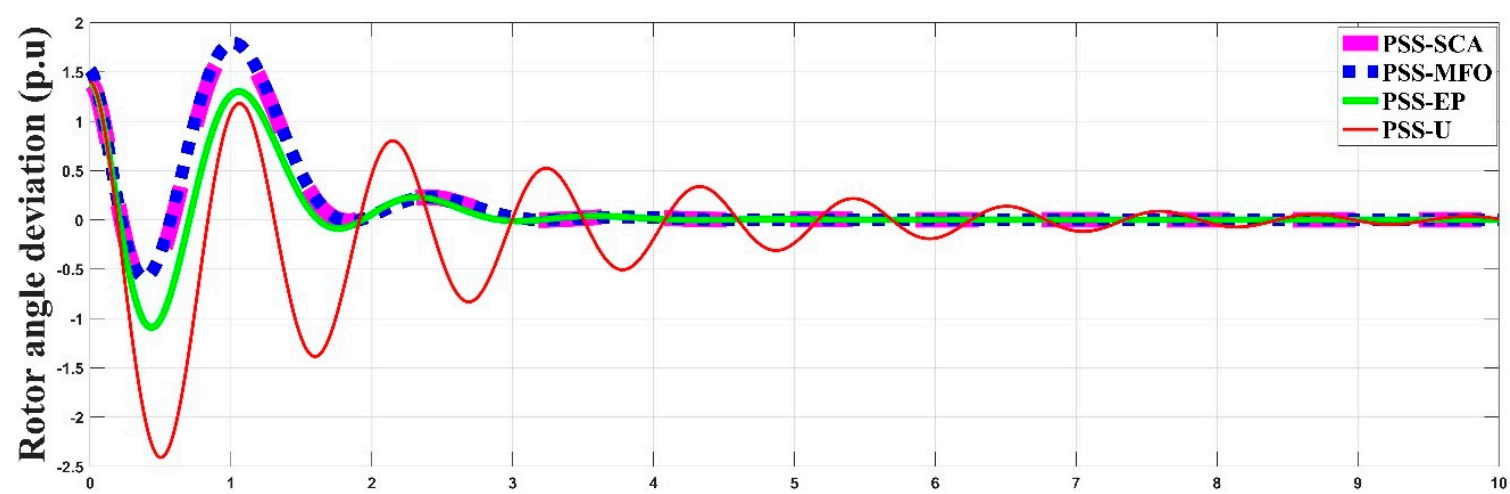

Time (s)

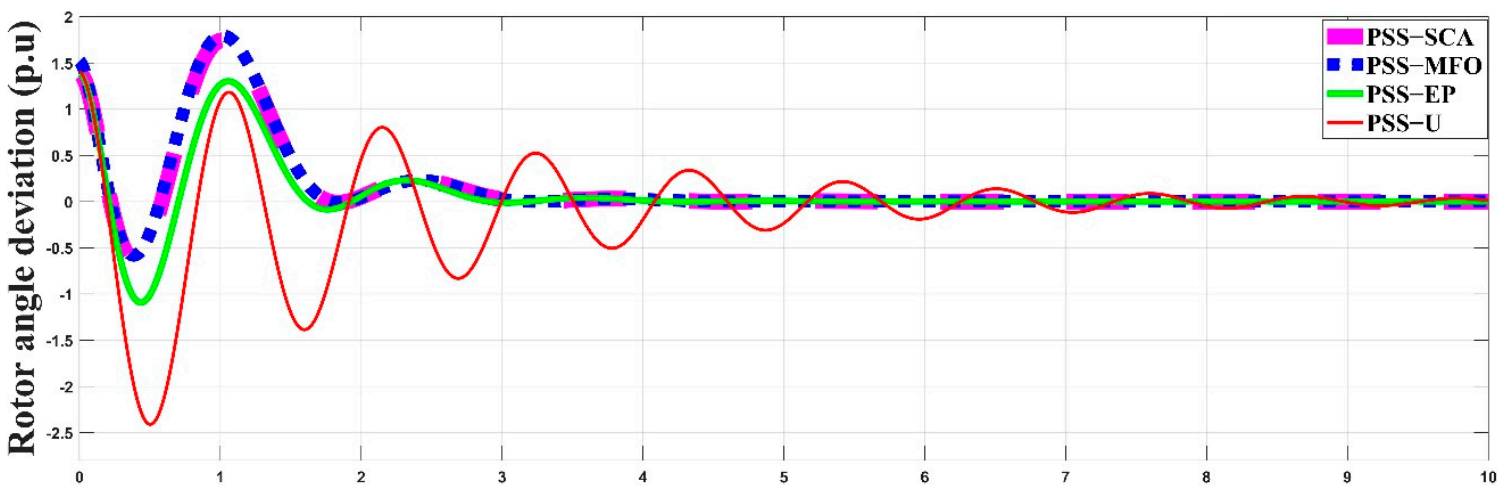

Time (s)

Figure 11. Rotor angle deviation response of PSS-SCA, PSS-MFO, PSS-EP, and PSS-U for case 3.

All eigenvalues are on the negative side of the complex s-plane that show the stable condition of all systems for case 3, as shown in Figure 12. The eigenvalue of PSS-SCA is on the left-most of the imaginary axis and is shifted towards the real axis of the s-plane as compared with those of the other techniques PSS-MFO, PSS-EP, and PSS-U.

Table 5 shows the PSS parameters, $T_{1}, T_{2}$ and $T_{\mathrm{W}}$, of the lead lag controller tuned by the three optimization techniques with the minimum objective function for case 3 .

Table 5. Comparison of PSS-SCA, PSS-MFO, PSS-EP and PSS-U for Case 3.

\begin{tabular}{ccccc}
\hline Type & $\boldsymbol{T}_{\boldsymbol{w}}$ & $\boldsymbol{T}_{1}$ & $\boldsymbol{T}_{2}$ & Objective Function \\
\hline PSS-SCA & 0.4858 & 3.8352 & 0.4601 & -1.4738 \\
PSS-MFO & 0.4783 & 3.9594 & 0.4618 & -1.4737 \\
PSS-EP & 0.6201 & 2.6964 & 0.3870 & -1.4012 \\
PSS-U & 1.5 & 0.2 & 0.05 & -0.4298 \\
\hline
\end{tabular}



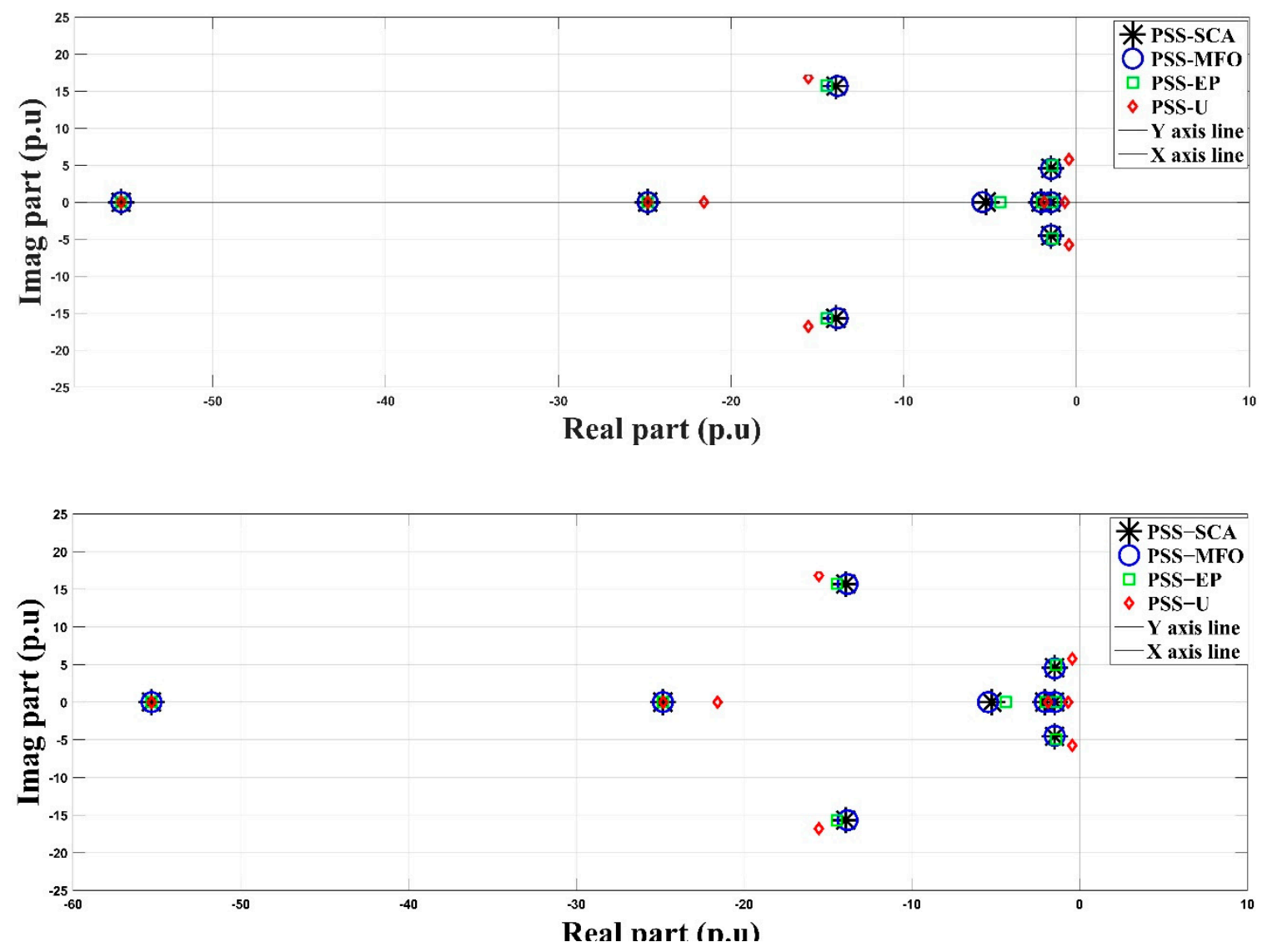

Figure 12. Eigenvalues distribution of PSS-SCA, PSS-MFO, PSS-EP, and PSS-U for case 3.

\section{Conclusions}

This study discussed modelling and optimization techniques for solving the problem related to small signal stability of an SMIB system and the effectiveness of the objective function in tuning the PSS lead lag controller parameters to improve the angle stability of an SMIB system. The power system stability efficiency improved by increasing the safety margin through the application of a maximum eigenvalue as the objective function. A linearized state space model was developed to determine the eigenvalues mode associated with low frequency oscillations. The application of optimization techniques moved the eigenvalues further towards the left side of the imaginary axis and towards the real axis line of the complex s-plane. Comparison of the optimization techniques based on three cases for the PSS lead lag controller using maximum eigenvalues as the objective functions revealed the PSS-SCA could enhance the safety margin and improve the stability performance. Compared with the MFO and EP techniques, the SCA optimization technique obtained better calculations of the optimal parameters to enhance the capability of the damping controller. From the perspective of iterations, EP converged with the fewest iterations, i.e., 16-30 iterations, followed by the SCA and MFO techniques that converged at 52-150 iterations. Despite the large iteration number, the number of iterations using the SCA approach was still minimal and acceptable. In conclusion, the SCA optimization technique obtained better calculations of the optimal parameters to enhance the capability of the damping controller compared to those achieved with the MFO and EP techniques. 
Author Contributions: Conceptualization, A.W.K. and N.A.M.K.; methodology, A.W.K. and N.A.M.K.; software, A.W.K.; validation, A.W.K. and N.A.M.K.; formal analysis, A.W.K.; investigation, A.W.K.; resources, A.W.K. and N.A.M.K.; data curation, A.W.K.; writing一original draft preparation, A.W.K.; writing-review and editing, A.W.K., N.A.M.K. and M.A.A.M.Z.; visualization, A.W.K. and N.A.M.K.; supervision, N.A.M.K. and M.A.A.M.Z.; project administration, A.W.K. and N.A.M.K.; funding acquisition, N.A.M.K. All authors have read and agreed to the published version of the manuscript.

Funding: The authors appreciate the support given by the Ministry of Higher Education (MOHE) and the Universiti Kebangsaan Malaysia (UKM) for the operational and financial support of this project under Project Code GUP-2020-018.

Institutional Review Board Statement: Not applicable.

Informed Consent Statement: Not applicable.

Data Availability Statement: Not applicable.

Conflicts of Interest: The authors declare no conflict of interest.

\section{References}

1. Kundur, P. Power System Stability and Control, 3rd ed.; McGraw-Hill Education: New York, NY, USA, $1994 ;$ pp. $17-817$.

2. Machowski, J.; Bialek, J.W.; Bumby, J.R. Power System Dynamics: Stability and Control, 2nd ed.; John Wiley and Sons Ltd.: Chichester, UK, 1997.

3. Anderson, P.M.; Fouad, A.A. Power System Control and Stability, 2nd ed.; Wiley-IEEE Press: Danvers, MA, USA, 2002.

4. Hannan, M.A.; Islam, N.N.; Mohamed, A.; Lipu, M.S.H.; Ker, P.J.; Rashid, M.M.; Shareef, H. Artificial intelligent based damping controller optimization for the multi-machine power system: A review. IEEE Access 2018, 6, 39574-39594. [CrossRef]

5. Kamari, N.A.M.; Musirin, I.; Ibrahim, A.A. Swarm intelligence approach for angle stability improvement of PSS and SVC-based SMIB. JEET 2020, 15, 1001-1014. [CrossRef]

6. Yang, M.; Cao, W.; Lin, T.; Zhao, J.; Li, W. Low frequency damping control for power electronics-based AC grid using inverters with built-in PSS. Energies 2021, 14, 2435. [CrossRef]

7. Wang, B.; Sun, K. Formulation and characterization of power system electromechanical oscillations. IEEE Trans. Power Syst. 2016, 31, 5082-5093. [CrossRef]

8. Eslami, M.; Shareef, H.; Mohamed, A. Application of artificial intelligent techniques in PSS design: A survey of the state-of-the-art methods. Prz. Elektrotechniczny 2011, 87, 188-197.

9. Zhang, Y.; Chen, G.P.; Malik, O.P.; Hope, G.S. An artificial neural network based adaptive power system stabilizer. IEEE Trans. Energy Convers. 1993, 8, 71-77. [CrossRef]

10. Li, C.; Deng, J.; Zhang, X.-P. Coordinated design and application of robust damping controllers for shunt FACTS devices to enhance small-single stability of large-scale power systems. JPES 2017, 3, 399-407.

11. Jiang, S.; Gole, A.M.; Annakkage, U.D.; Jacobson, D.A. Damping performance analysis of IPFC and UPFC controllers using validated small-single models. IEEE Trans. Power Deliv. 2011, 26, 446-454. [CrossRef]

12. Parastvand, H.; Bass, O.; Masoum, M.A.S.; Chapman, A.; Lachowicz, L. Cyber-security constrained placement of FACTS devices in power networks from a novel topological perspective. IEEE Access 2020, 8, 108201-108215. [CrossRef]

13. Zhou, L.; Swain, A.; Ukil, A. Reinforcement learning controllers for enhancement of low voltage ride through capability in hybrid power systems. IEEE Trans. Ind. Inform. 2020, 16, 5023-5031. [CrossRef]

14. Guangzheng, Y.; Lin, T.; Zhang, J.; Tian, Y.; Yang, X. Coordination of PSS and FACTS damping controllers to improve small signal stability of large-scale power system. JPES 2019, 5, 507-514.

15. Zuo, J.; Li, Y.; Shi, D.; Duan, X. Simultaneous robust coordinated damping control of power system stabilizers (PSSs), static var compensator (SVC) and doubly-fed induction generator power oscillation dampers (DFIG PODs) in multimachine power systems. Energies 2017, 10, 565. [CrossRef]

16. Baek, S.-M.; Park, J.-W. Nonlinear parameter optimization of FACTS controller via real-time digital simulator. IEEE Trans. Ind. Appl. 2013, 49, 2271-2278. [CrossRef]

17. Al-Ismail, F.S.; Hassan, M.A.; Abido, M.A. RTDS implementation of STATCOM-based power system stabilizers. CJECE 2014, 37, 48-56. [CrossRef]

18. Pandey, R.K.; Gupta, D.K. Integrated multi-stage LQR power oscillation damping FACTS controller. JPES 2018, 4, 83-92. [CrossRef]

19. Jiang, P.; Fan, Z.; Feng, S.; Wu, X.; Cai, H.; Xie, Z. Mitigation of power forced oscillations based on unified power flow controller. JMPSCE 2019, 7, 99-112. [CrossRef]

20. Quan, W.; Su, Q. Improved adaptive backstepping approach on STATCOM controller of nonlinear power system. IEEE Access 2019, 7, 139109-139117. [CrossRef]

21. Asgharian, A.; Tavakoli, S.A. A systematic approach to performance weights selection in design of robust H PSS using genetic algorithms. IEEE Trans. Energy Convers. 1996, 11, 111-117. [CrossRef] 
22. Kahouli, A.; Guesmi, T.; Abdallah, H.H.; Quali, A. A genetic algorithm PSS and AVR controller for electrical power system stability. In Proceedings of the 6th International Multi-Conference on Systems, Signals and Devices, Djerba, Tunisia, 23-26 March 2009.

23. Hassan, L.H.; Moghavvemi, M.; Almurib, H.A.F.; Muttaqi, K.M. A coordinated design of PSSs and UPFC-based stabilizer using genetic algorithm. IEEE Trans. Ind. Appl. 2014, 50, 2957-2966. [CrossRef]

24. Gupta, N.; Agarwal, T. Design of optimal power system stabilizer using GA and PSO: A comparative study. In Proceedings of the IEEE International Conference on Recent Advances and Innovations in Engineering, Jaipur, India, 23-25 December 2016.

25. Verdejo, H.; Pino, V.; Kliemann, W.; Becker, C.; Delpiano, J. Implementation of particle swarm optimization (PSO) algorithm for tuning of power system stabilizers in multimachine electric power systems. Energies 2020, 13, 2093. [CrossRef]

26. Islam, N.N.; Hannan, M.A.; Shareef, H.; Mohamed, A. Power system stabilizer design using BAT optimization algorithm in multimachine power system. In Proceeding of the IEEE Student Conference on Research and Development, Putrajaya, Malaysia, 16-17 December 2013.

27. Sheshnarayan; Verma, B.; Padhy, P.K. Design PID Controller based PSS using cuckoo search optimization technique. In Proceedings of the 4th International Conference on Recent Trend on Electronics, Information, Communication \& Technology, Bengaluru, India, 17-18 May 2019.

28. Islam, N.N.; Hannan, M.A.; Shareef, H.; Mohamed, A. SVC Damping controller design based on firefly optimization algorithm in multi machine power system. In Proceedings of the IEEE Conference on Clean Energy and Technology, Langkawi, Malaysia, 18-20 November 2013.

29. Verdejo, H.; Torres, R.; Pino, V.; Kliemann, W.; Becker, C.; Delpiano, J. Tuning of controllers in power systems using a heuristicstochastic approach. Energies 2019, 12, 2325. [CrossRef]

30. Kamari, N.A.M.; Musirin, I.; Hamid, Z.A.; Rahim, M.N.A. EP-based optimisation for estimating synchronising and damping torque coefficients. AJBAS 2010, 4, 3741-3754.

31. Mirjalili, S. SCA: A sine cosine algorithm for solving optimization problems. Knowl. Syst. 2016, 96, 120-133. [CrossRef]

32. Mirjalili, S. Moth-flame optimization algorithm: A novel nature-inspired heuristic paradigm. Knowl. Syst. 2015, 89, 228-249. [CrossRef] 